02

\title{
Особенности распределения спектральных параметров межмолекулярных колебаний в воде, полученных с помощью КРС
}

\author{
(C) A.В. Крайский ${ }^{1}$, Н.Н. Мельник ${ }^{1}$, А.А. Крайский ${ }^{2}$ \\ ${ }^{1}$ Физический институт им. П.Н. Лебедева РАН, \\ 119991 Москва, Россия \\ ${ }^{2}$ Институт общей фризики им. А.М. Прохорова РАН, \\ 119991 Москва, Россия \\ e-mail: kraiski@sci.lebedev.ru
}

Поступила в редакцию 26.11.2018 г.

В окончательной редакции 03.07.2019 г.

Принята к публикации 21.10.2019 г.

С помощью комбинационного рассеяния света (КРС) исследованы спектральные характеристики межмолекулярных колебаний воды, связанные с низкочастотными (около $50 \mathrm{~cm}^{-1}$ в ИК поглощении) и с высокочастотными $\left(183.4 \mathrm{~cm}^{-1}\right.$ в ИК поглощении) колебаниями молекул воды. Рассмотрение проводилось в рамках представлений о классическом затухающем осцилляторе. В координатах квадрат ширины квадрат частоты для любого колебания характеристики его полосы отображаются в виде точки. Показано, что совокупность экспериментально полученных точек распределяется в некоторой области этой системы координат неравномерно. Обнаружено, что для каждого из колебаний внутри соответствующей области точки расположены на двух близких участках, разделенных практически незанятой точками полосой. Математическая обработка позволила показать наличие мелкомасштабной неоднородности распределения точек. Вопрос о том, является ли обнаруженная неоднородность спектральных параметров отражением реально существующих состояний в воде, требует дальнейшего исследования.

Ключевые слова: комбинационное рассеяние, вода, низкочастотные спектры, собственная частота, динамическая восприимчивость, спектральные координаты, неоднородное распределение.

DOI: $10.21883 /$ OS.2020.02.48960.339-18

\section{1. Введение}

Настоящая работа является продолжением работы [1] по исследованию низкочастотных (НЧ) спектров комбинационного рассеяния (КР) воды и слабых водных растворов. Она основывается на представленном там новом, разработанном нами методе исследования взаимозависимостей между положением спектральных максимумов и шириной лоренцианов, описывающих спектр колебательных линий динамической восприимчивости. Важность исследования этих спектров достаточно очевидна вследствие важной роли воды и водных растворов и обосновывается в [1] и в цитированной там литературе.

\section{2. Низкочастотные спектры воды и их особенностях}

Низкочастотный спектр КР воды достаточно хорошо известен [1-3]. Поскольку в нем отражаются свойства как среды, так и процесса рассеяния, то для описания свойств среды вводится преобразование спектра - так называемая редукция [4-9]. Более подробно этот вопрос изложен в [10]. Это преобразование сводится к умноже- нию интенсивности КР $I(v)$ на 2 множителя:

$$
\chi^{\prime \prime}(v)=\left(v_{i}-v\right)^{-4}[n(v)+1]^{-1} I(v),
$$

где $n(v)=[\exp (h v / k T)-1]^{-1}-$ бозе-эйнштейновский множитель, $v_{i}$ - частота возбуждающего лазерного излучения в $\mathrm{cm}^{-1}, T-$ температура, $\left(v_{i}-v\right)^{-4}-$ релеевский множитель. Полученную так величину $\chi^{\prime \prime}$ называют динамической восприимчивостью (ДВ). Достаточно широко употребляется название редуцированная или R-функция. B спектре динамической восприимчивости в области низких частот наблюдаются две характерные полосы, а в низкочастотной части низкочастотной полосы - характерный излом. Интерпретация этих полос подробно обсуждается в $[8,15]$.

В [11] предложено объяснение высокочастотной полосы $\left(183.4 \mathrm{~cm}^{-1}\right.$ в ИК поглощении) движением молекул вдоль направления соединяющей их водородной связи (мода растяжения связи), низкочастотной полосы (около $50 \mathrm{~cm}^{-1}$ в ИК поглощении) изгибным колебанием водородной связи. Эта интерпретация приведена и в [12], и с ней согласуются выводы в работах [13-15]. В [12] приводятся и другие объяснения причин появления этих полос. В работах [16-19] делается вывод, что обе полосы связаны не с колебанием водородной связи двух молекул, а с движением молекул в ограниченном пространстве из-за взаимодействия с окружающими молекулами [16-18]. В работах [20,9] высокочастотная полоса 
интерпретируется как колебание, связанное с изменением длины связи, а низкочастотная полоса определяется в [20] взаимодействием с соседями, а в [9] — частично изгибными колебаниями и частично движением в поле соседей. В [21] такой механизм предложен для обеих полос. В [22] для объяснения высокочастотной полосы предлагается кроме колебательного движения вдоль связи (нежесткий диполь) еще и упругая переориентация жестких диполей водородной связи. В отношении же низкочастотной полосы делается предположение о влиянии изгибных колебаний водородной связи.

Таким образом, для структуры колебаний межмолекулярных взаимодействий пока единой картины нет.

\section{3. Аппроксимирующая спектр функция}

При подборе аппроксимирующей спектр функции мы руководствовались следующими соображениями. Наиболее близкий к нашим условиям подход содержался в [9]. Этот принцип расчета и был взят за основу. Подробности описаны в $[2,23,24]$. При построении аппроксимирующей спектр функции мы исходили из представлений (например [25]), что в воде имеется некая большая структура более или менее связанных водородными связями молекул. В такой динамической системе имеются большие наборы собственных частот, которые и могут наблюдаться в оптических экспериментах. Представляется вполне очевидным, что они могут группироваться в виде наблюдающихся двух полос. Водородные связи находятся в некотором динамическом равновесии: некоторое время живут, затем рвутся, затем опять могут устанавливаться с теми же или с другими молекулами. При этом в среде постоянно имеются некоторые движения, некоторые неоднородности и т.П. Поэтому мы видим в спектре отражение статистической картины. При КР этой средой одномоментно поглощается фотон возбуждающего излучения, испускается стоксов фотон и рождается затухающее колебание среды (т.е. свободное колебание) на одной из частот, близкой к одной из собственных частот среды. Поэтому спектр наблюдаемого рассеяния связывается со спектром рождающихся свободных колебаний среды. Такой спектр описывается функцией Лоренца. Фактически мы ввели гипотезу о представлении колебаний в виде возбуждаемого приложением импульса свободно затухающего (если так можно выразиться) осциллятора.

Излом на низкочастотной полосе обычно связывается с медленным хаотическим поворотным движением молекулы в поле своих соседей $[9,12]$. Напомним, что в [9] это движение связывается частично с низкочастотной полосой. В [1-3] аппроксимирующую функцию для ДВ $\chi^{\prime \prime}$ мы записывали в виде

$$
\begin{aligned}
\chi^{\prime \prime}= & \frac{I_{r} v}{1+\left(2 v / \delta v_{r}\right)^{2}}+\frac{I_{\text {low }}}{1+\left(2\left(v-v_{\text {low }}\right) / \delta v_{\text {low }}\right)^{2}} \\
& +\frac{I_{h}}{1+\left(2\left(v-v_{h}\right) / \delta v_{h}\right)^{2}}+I_{c} v,
\end{aligned}
$$

где 9 подгоночных параметров $I_{r}, \delta v_{r}, I_{\text {low }}, v_{\text {low }}, \delta v_{\text {low }}$, $I_{h}, v_{h}, \delta v_{h}, I_{c}$. В (2) 2-е и 3-е слагаемые - соответственно низкочастотный (около $50 \mathrm{~cm}^{-1}$ ) и высокочастотный (около $180 \mathrm{~cm}^{-1}$ ) лоренцианы, характеризующиеся интенсивностью $I$, положением максимума $v$ и шириной $\delta v$ с соответствующими нижними индексами. Первым слагаемым мы описываем хаотическое поворотное движение молекулы, последнее слагаемое связано с постоянным фоном в спектре КР (мы его относим к люминесценции, это позволило получать хорошую аппроксимацию до $320 \mathrm{~cm}^{-1}$ ) [1-3]. Эти спектральные параметры определялись подгонкой кривой, вычисляемой по формуле (2), по методу наименьших квадратов к спектру ДВ в диапазоне частот $4-320 \mathrm{~cm}^{-1}$ [1-3]. В процессе наших исследований было выявлено, что кроме направленных изменений параметров, связанных, например, с изменением концентрации перекиси водорода в воде, наблюдаются значительные случайные изменения всех спектральных параметров [1-3].

Обнаружено, что эти изменения и в воде, и в растворе перекиси водорода для обоих лоренцианов оказались в значительной степени коррелированы для положения максимумов и ширин линий каждого из лоренцианов в отдельности $[1,3,24]$. Обсуждения вопросов взаимной корреляции спектральных параметров лоренциана, описывающего межмолекулярные колебания воды и слабых водных растворов, в литературе не встречались. Так что в [3,24], видимо, впервые приведен факт наблюдения такой корреляции. В [1] же впервые в печатной статье приводится интерпретация этой взаимной корреляции спектральных параметров на основе свойств классического осциллятора с основными (вытекающими из этих свойств) следствиями. В литературе мы не нашли указаний на эту особенность спектральных параметров. В [1] было показано, что для классического осциллятора при изменяющемся затухании и неизменной собственной частоте (СЧ) в координатах квадрат измеряемой ширины контура - квадрат положения максимума этого контура (далее будем называть их частотными координатами) должна наблюдаться линейная зависимость с отрицательным коэффициентом. Точка пересечения этой прямой с осью квадрата частот определяется собственной частотой максимума поглощения, а угловой наклон должен быть равен 0.5. Меньшая величина этого наклона связана с неоднородным уширением линии. Отсюда можно определить однородную ширину линии и, следовательно, оценить время затухания колебания. Действительно, определенные так собственные частоты для обоих лоренцианов хорошо совпали с известными значениями линий ИК поглощения [1]. Мы считаем, что это является достаточно хорошим аргументом в пользу верности принятых нами представлений.

Отметим, что таким образом мы исходя только из свойств наблюдаемых параметров спектра, частоты максимума и ширины линии определяем другие характеристики среды, ранее не определявшиеся с помощью КРС: собственную частоту колебаний и однородную ширину 
полосы. Вследствие линейности зависимости квадрата положения максимума лоренциана от квадрата наблюдаемой ширины при постоянной собственной частоте колебаний и изменяющемся затухании осциллятора эти координаты являются предпочтительными при обработке данных.

В [1] для воды были проведены 2 серии записи спектров. В первой серии было записано 3 спектра, во второй - 4 спектра. Спектры в каждой из серий записывались в один и тот же день, а интервал между сериями составлял много месяцев. В частотных координатах для каждой из серий в отдельности нельзя было построить описанную выше линейную зависимость, но общая совокупность хорошо аппроксимируется линейной зависимостью. В связи с этим возникает вопрос, как будут выглядеть в частотных координатах данные при большем количестве точек и какую информацию об объекте исследования оттуда можно получить.

Цель настоящей работы - обработать большое количество (несколько десятков) спектров одного образца, представить их в частотных координатах, исследовать особенности их расположения в этих координатах и выяснить, насколько это расположение соответствует модели классического затухающего осциллятора.

\section{4. Полученные распределения спектральных параметров в частотных координатах, собственные частоты и коэффициенты неоднородности всей совокупности}

В работе мы представляем в частотных координатах полученные данные для обоих колебаний, и на примере высокочастотного лоренциана представляем одну из методик анализа полученных результатов.

Методика измерений полностью описана в [1-3]. Отметим только, что записывались деполяризованные спектры. Измерения проводились в одной и той же запаянной ампуле медицинской воды для инъекций. Было проведено 5 серий измерений и записан в общей совокупности 51 спектр. Спектры каждой серии записывались в течение одного дня.

На рис. 1 показаны наборы экспериментальных точек в частотных координатах для обоих колебаний, которые неплохо аппроксимируются линейными зависимостями. Графики сильно растянуты по оси ординат: разброс по этой оси для высокочастотного колебания составляет менее $10 \%$ по отношению к среднему значению частоты, а для низкочастотного колебания - менее $15 \%$. По параметрам аппроксимирующей прямой можно оценить эффективные значения СЧ колебаний и коэффициентов неоднородного уширения по методу, описанному в [1]. Для высокочастотного колебания эти величины составляют $184.3 \mathrm{~cm}^{-1}$ для СЧ и 1.65 для коэффициента неоднородности (отношение наблюдаемой ширины лоренциана к однородной ширине), что неплохо совпадает со значениями $184.6 \mathrm{~cm}^{-1}$ и 1.67 , полученными на серии из 7 точек в [1]. Для низкочастотного колебания соответствующие значения, полученные по совокупности 51 точки, составляют $49.8 \mathrm{~cm}^{-1}$ для СЧ и 4.15 для коэффициента неоднородности, что также неплохо совпадает с данными, полученными в [1] по 7 точкам: $49.9 \mathrm{~cm}^{-1}$ и 4.39 соответственно. Для коэффициента неоднородности низкочастотного колебания расхождение составляет лишь около $5 \%$, для СЧ - $1 \%$, для высокочастотного колебания расхождение значительно меньше: $0.2 \%$ для обеих величин. Отметим, что в большом наборе данных по обеим координатам больший разброс: стандартное отклонение (CO) для наблюдаемых квадратов ширин составляет $1400 \mathrm{~cm}^{-2}$ против $949 \mathrm{~cm}^{-2}$ для 7 точек в [1], а для квадратов частот $-513 \mathrm{~cm}^{-2}$ против $219 \mathrm{~cm}^{-2}$ для 7 точек.

Таким образом, значения средних параметров прямых, аппроксимирующих большую совокупность точек (51 точка, настоящая работа) и малую (7 точек, [1]), близки между собой.

\section{5. Проявления неоднородности распределения спектральных параметров в частотных координатах}

Особенность представленных экспериментальных точек состоит в том, что на графике выше средней аппроксимирующей прямой имеется область с отсутствием экспериментальных точек (далее будем называть пустой областью). Эта область имеет вид полосы с почти параллельными сторонами, практически параллельными аппроксимирующей прямой. Нижний ее край близок к этой прямой и лежит чуть выше ее. Ширина этой полосы составляет около $10 \%$ всей ширины распределения. Полоса имеет открытый левый верхний край при значении квадрата ширин около $27000 \mathrm{~cm}^{-2}$ и как бы замыкается группой точек на правом нижнем крае вблизи значения по оси абсцисс около $31000 \mathrm{~cm}^{-2}$. Выше пустой области находится 15 точек. Таким образом, явно видна некоторая структурированность данных в частотных координатах. Наличие пустой области свидетельствует, что вероятность попадания в нее точек мала. Поскольку площадь этой области на взгляд можно оценить менее $10 \%$ от площади, занимаемой всей совокупностью точек, то вероятность попадания в нее при 51 точке составляет не более 0.2. При этом в окрестности аппроксимирующей прямой (чуть ниже ее) наблюдается очень большая плотность точек, что выражается в перекрытии маркеров в нескольких группах. Визуально складывается и впечатление о более мелкомасштабной неоднородности как в направлении, перпендикулярном аппроксимирующей прямой, так и вдоль ее направления.

На рис. $1, b$ показана в частотных координатах совокупность данных для низкочастотного колебания. Здесь также наблюдается подобная незаполненная область. 

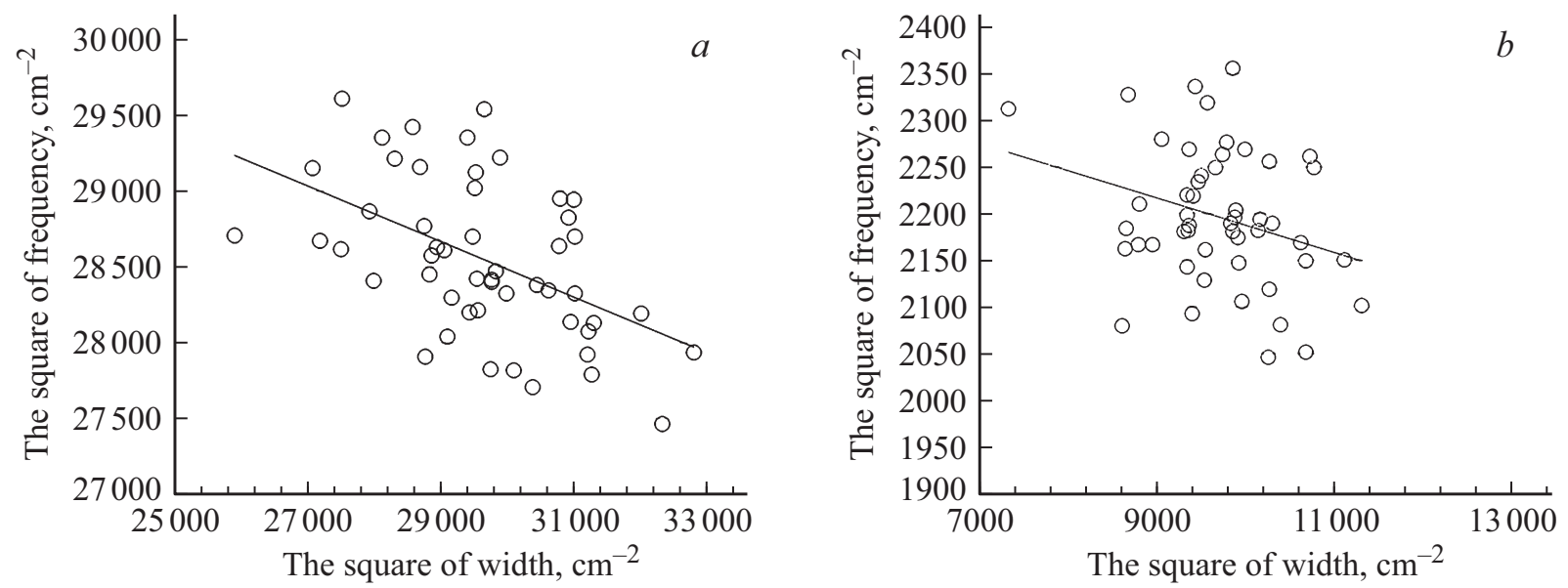

Рис. 1. (a) Зависимости квадратов положения максимума высокочастотного лоренциана от квадрата наблюдаемой его ширины и их аппроксимация зависимостью $y=-0.183 x+33972$ (сплошная прямая). (b) Зависимости квадратов положения максимума низкочастотного лоренциана от квадрата наблюдаемой его ширины и их аппроксимация зависимостью $y=-0.029 x+2478$ (сплошная прямая).

Выше этой области лежат 14 точек. Здесь верхние и нижние точки также соединяются узкой перемычкой в виде узкой протяженной искривленной совокупности точек с повышенной плотностью, но эта перемычка расположена в центре картины. Правее по аппроксимирующей прямой наблюдаются еще две близкие неоднородности. В этой работе на этом мы закончим рассмотрение особенностей низкочастотного колебания и сосредоточимся на дальнейшем рассмотрении особенностей высокочастотного колебания.

На рис. 2, $a$ к точкам, показанным на рис. 1, $a$, добавлены 7 точек для спектров, полученных в [1], которые обозначены крупными косыми крестами. Видно, что они также образуют подобную структуру, положение которой практически совпадает с областью большого набора точек, и расположены вблизи обеих границ пустой зоны. Таким образом, можно сделать вывод, что в этой части всей области имеются участки с достаточно высокой вероятностью попадания точек. Подобная же картина наблюдается и для НЧ колебания..

Важно то, что пустая область образуется в одном и том же месте в трех разных экспериментах. Следует иметь в виду, что измерения большой серии проводились на другом образце, нежели образцы малой серии. Таким образом, можно утверждать, что со значительной степенью вероятности распределение точек в частотных координатах параметров для высокочастотного колебания воды сильно неоднородно, и внутри области, занимаемой экспериментальными точками, имеется значительная область, вероятность попадания точек в которую мала.

При более внимательном рассмотрении можно увидеть и другие проявления неоднородности (рис. 1, $a$, 2, a). На рис. 2, a показаны проведенные нами разбиения точек на группы, которые аппроксимированы прямыми линиями (ниже описано, каким способом было получено это разбиение). Сразу следует отметить, что это разбие- ние не однозначно (особенно для точек, лежащих ниже пустой зоны), возможны различные способы, и полученные результаты для молекулярных постоянных могут быть различными. Поэтому необходимо максимальным образом объективизировать этот процесс.

На рис. 2, $b$ показаны аппроксимации модельных групп точек, построенных с помощью генератора случайных чисел и разбитых на группы таким же способом. Самым простым способом совокупность экспериментальных точек (рис. $1, a, 2, b)$ можно разбить на 2 области: выше области с низкой вероятностью попадания точек (пустой области) и ниже этой области.

Аппроксимрующие средние прямые этих областей описываются следующими уравнениями: $y=-0.1697 x+34163$ для верхней совокупности и $y=-0.1695 x+33330$ для нижней. Замечательно, что эти прямые практически параллельны, различие в угловых наклонах порядка $0.01 \%$. Эффективные коэффициенты неоднородности [1] этих совокупностей также практически не отличаются: 1.717 для верхней и 1.718 для нижней совокупности. Эффективные СЧ составляют 184.8 и $182.6 \mathrm{~cm}^{-1}$. Несмотря на то, что аппроксимирующие линии верхней и нижней совокупностей параллельны, аппроксимирующая всю совокупность точек прямая не параллельна им. Связано это с тем, что центры тяжести этих совокупностей смещены вдоль аппроксимирующей линии. Следует отметить, что при этом анализе мы использовали только прямо полученные результаты из аппроксимации спектров ДВ с помощью выражения (2), они не подвергались сложным перерасчетам, и не использовалась никакая субъективная фильтрация данных. На основании различия эффективных СЧ этих совокупностей, можно утверждать, что состояния воды, в которых записывались соответствующие спектры, различаются по своим свойствам. О причинах этого мы в настоящий момент ничего определенного сказать не можем. 
$a$
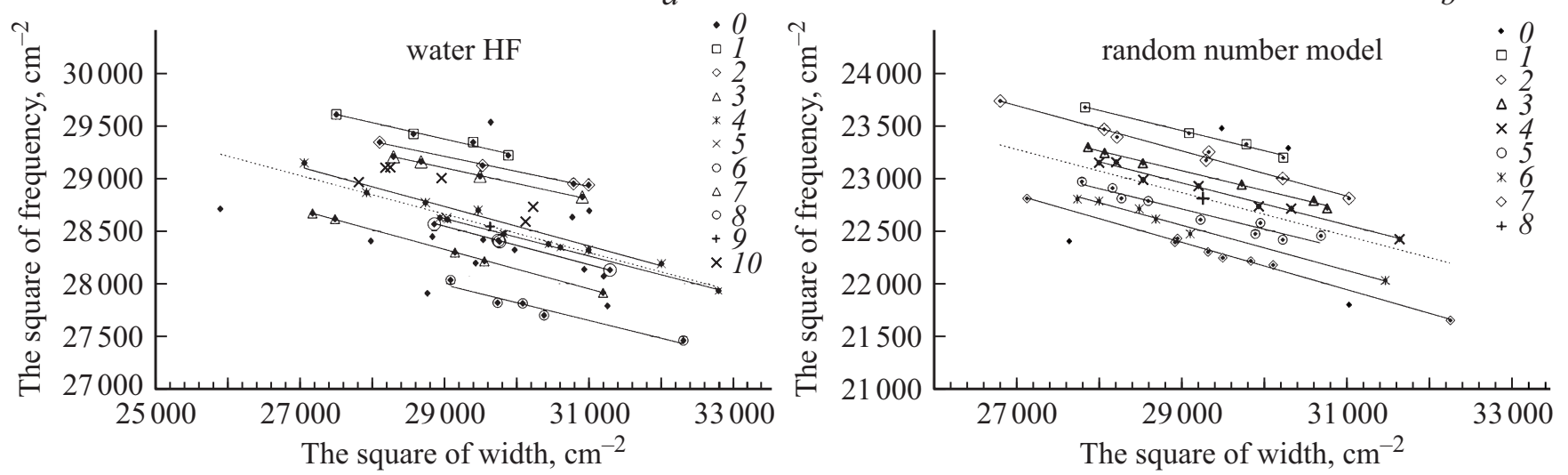

Рис. 2. Точки серии 0 означают всю совокупность точек. (a) Зависимости квадратов положения максимума высокочастотного лоренциана от квадрата наблюдаемой его ширины и их аппроксимация зависимостью $y=-0.183 x+33972$ (щтриховая прямая) и разбиение на группы по расстоянию от этой прямой. Точка $9-$ центр тяжести всей совокупности, точки $10-$ эксперимент [1]. (b) Такая же зависимость для модели случайных чисел. Аппроксимация всей совокупности прямой $y=-0.208 x+28884$ (штриховая прямая), точка 8 - центр тяжести всей совокупности.

Параметры осцилляторов для групп точек на рис. 2, a

\begin{tabular}{c|c|c|c|c|c}
\hline $\begin{array}{c}\text { Номер } \\
\text { группы }\end{array}$ & $\begin{array}{c}\text { Угловой } \\
\text { коэффициент }\end{array}$ & $\begin{array}{c}\text { Коэффициент } \\
\text { неоднородности }\end{array}$ & $\begin{array}{c}\text { Собственная } \\
\text { частота, } \mathrm{cm}^{-1}\end{array}$ & $\begin{array}{c}\text { Однородная } \\
\text { ширина, } \mathrm{cm}^{-1}\end{array}$ & $\begin{array}{c}\text { Время } \\
\text { затухания, fs }\end{array}$ \\
\hline 1 & -0.154 & 1.802 & 184.0 & 95.1 & 77.3 \\
2 & -0.143 & 1.867 & 182.7 & 92.3 & 79.7 \\
3 & -0.149 & 1.834 & 182.8 & 105.3 & 78.8 \\
4 & -0.187 & 1.633 & 184.9 & 103.6 & 71.1 \\
5 & -0.178 & 1.675 & 183.8 & 104.0 & 70.7 \\
6 & -0.182 & 1.658 & 183.8 & 104.0 & 70.8 \\
7 & -0.188 & 1.631 & 184.1 & 108.7 & 67.7 \\
10 & -0.201 & 1.577 & 184.3 & 104.1 & 70.6
\end{tabular}

Экспериментальные точки имеют значительный разброс как в направлении, перпендикулярном к аппроксимирующей всю совокупность прямой, так и вдоль ее направления. При постоянной для одной группы точек СЧ осцилляторов разброс вдоль направления прямой связан с изменениями затухания осциллятора, а разброс в перпендикулярном направлении - с возможным изменением СЧ осцилляторов. Поэтому представляется необходимым проанализировать эти распределения.

\section{6. Анализ неоднородности распределения спектральных параметров в частотных координатах}

Идея анализа достаточно проста - нам надо найти совокупности точек, через которые можно провести прямые. Сложность состоит в том, что при такой совокупности точек можно провести прямые с различающимися угловыми коэффициентами. Отметим, что выбранные координаты образуют евклидово пространство, и здесь можно пользоваться геометрическими представлениями. На первом этапе представляется очевидным в качестве опорной линии взять линию, аппроксимирующую всю совокупность точек. При этом будем считать положительным расстояние до этой линии для точек, расположенных выше прямой, для расположенных ниже - отрицательным. Рассчитаем расстояние от каждой точки до аппроксимирующей прямой и построим их упорядоченную по убыванию расстояний последовательность. Такая последовательность показана на рис. 3, a (кривая 1). Здесь по оси ординат отложено расстояние до прямой, по оси абсцисс - номер точки в последовательности.

Если точки расположены на линейной зависимости, параллельной выбранной прямой, то расстояния одинаковы, и на этой последовательности они будут расположены горизонтально. Для другой группы точек, также лежащих на параллельной прямой на другом расстоянии, в последовательности также образуется свой горизонтальный участок. Если точки этих участков расположены в упорядоченной последовательности рядом, то в распределении получится два горизонтальных участка на разных уровнях, т.е. образуется разрыв. Если между 

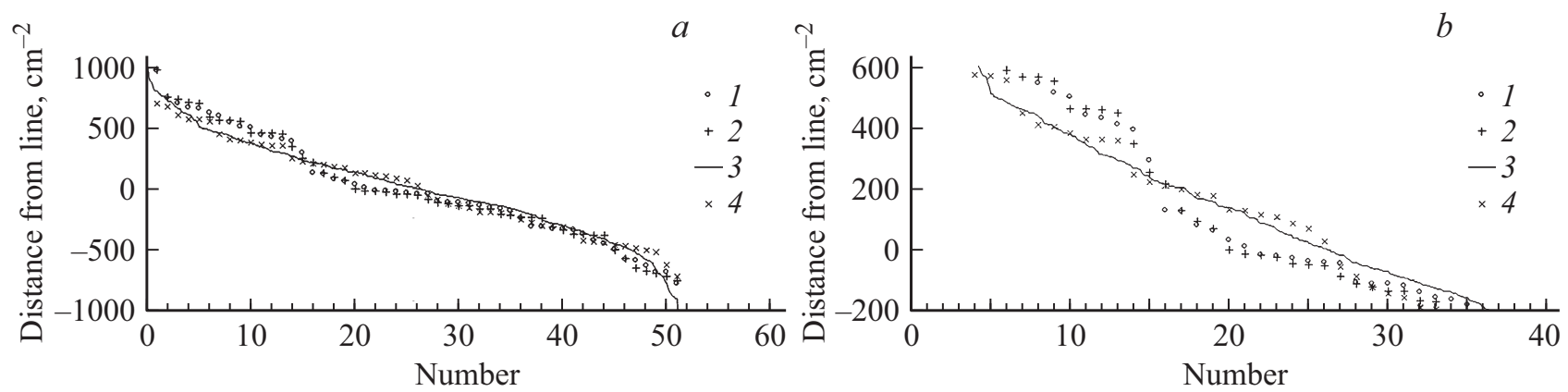

Рис. 3. (а) Упорядоченные по убыванию расстояния от точек в частотных координатах до прямой: $(1)$ для экспериментальных точек и прямой с наклоном 0.183; (2) для экспериментальных точек и прямой с наклоном 0149; (3) точек случайной последовательности объемом 510 точек; (4) точек случайной последовательности объемом 51 точка. $(b)$ Фрагмент зависимостей рисунка $a$. Меньшим номерам по оси абсцисс в упорядоченной по расстояниям последовательности соответствуют верхние точки на рис. 2,a.

точками таких участков расположены другие точки, то будет некоторая переходная область с наклоном. В реальном эксперименте вероятность одинакового расстояния мала, и будет наблюдаться наклон тем меньший, чем больше вероятность попадания точек в некоторый интервал Можно говорить о плотности точек на единичный интервал расстояния (здесь в точках $/ \mathrm{cm}^{-2}$ ), что удобно для сопоставления разных участков. Для сопоставления разных экспериментов удобно вводить нормированную на число точек величину. Эта величина обратно пропорциональна производной упорядоченного распределения, и при нормировке на интеграл по всему интервалу расположения точек ее можно назвать плотностью вероятности попадания на интервал. Интеграл от нее по какому-либо интервалу расстояний показывает вероятность попадания в этот интервал.

Если прямую, до которой мы рассчитываем расстояние от точки, мы начнем поворачивать вокруг точки, лежащей на этой прямой (здесь это центр тяжести всех точек, и эту точку прямой мы будем называть центром), то расстояния до точек начнут изменяться в зависимости от угла поворота и расстояния точки от центра. Соответствующие упорядоченные по убыванию распределения для нового выбранного положения начнут изменяться, и прежние горизонтальные участки могут стать негоризонтальными. При этом точки, относящиеся к какому-либо спектру, могут изменять свои номера в последовательности. Однако некоторые точки могут попасть на другую прямую, параллельную новому выбранному направлению.

Два, полученные так упорядоченные распределения для двух опорных прямых, показаны на рис. 3. Здесь не наблюдаются горизонтальные участки, но волнообразная картина с участками в виде наклонных прямых с разрывами между ними или в виде участка с переменным наклоном и изломом при переходе к соседнему явно видна. Кривая 1 (пустые кружки) построена для расстояний до прямой, аппроксимирующей всю совокупность точек (наклон 0.183). Для первых точек $(1-14)$ по оси абсцисс видны участки с чередующимися по величине наклонами, но без горизонтальных участков, что лучше видно на рис. $3, b$. Потом наблюдается большое падение уровня к точке 16 почти на $300 \mathrm{~cm}^{-2}$, связанное с переходом через область с низкой вероятностью. Затем наблюдаются малые скачки между горизонтальными участками (точки 27-28) и после горизонтального участка 31-32. Для кривой же 2 на тех же рисунках (прямые кресты, расстояние до прямой, проходящей через центр с наклоном 0.149) картина обратная: на тех же участках в интервале 1-13 наклоны меньшие с более четко выраженными разрывами на границах участках, переход через пустую область менее четко выражен и происходит от точки 13 к 17 с изменением уровня на $330 \mathrm{~cm}^{-2}$. Это свидетельствует о том, что первые 3 участка имеют наклон ближе к 0.149, а для границ пустой области наклоны ближе к 0.183. Для кривой 2 (прямые кресты) точка 27 приблизилась к точке 28, сгладив разрыв, наклоны участков, содержащих эти точки, больше, что также свидетельствует о том, что наклон для прямых, связанных с этими точками, ближе к 0.183.

По разрывам на распределениях мы выделили 8 участков. Эти участки и их аппроксимация прямыми линиями показаны на рис. 2, $a$.

Параметры, полученные для всех участков, показаны в таблице. В группе 0 показаны эффективные данные для всей совокупности 51 точек. Первые 3 участка относятся к верхней области. Хотя разбиение на группы проводилось по расстояниям до прямой с наклоном -0.183 , эти три участка при линейной аппроксимации имеют другой, свой собственный наклон, близкий к -0.149 . Видно, что коэффициенты неоднородности для них больше, чем для нижней области и заметно отличаются от общего для всех 51 точек коэффициента. Все эти 3 коэффициента близки по значениям и меньше, чем угловой коэффициент общей совокупности этих трех групп, приведенный в конце раздела 5. Это связано с тем, что каждая, более высоко расположенная группа точек сдвинута в сторону меньших ширин. Коэффициент неоднородности, однородная ширина и время затухания в значительной 
степени определяются угловым наклоном, и поэтому также различны для групп верхней и нижней совокупностей точек. Таким образом, для групп верхней совокупности по сравнению с группами нижней коэффициент неоднородности больше, однородная ширина меньше и время затухания больше. Собственные частоты слабо различаются между группами. Следует отметить, что разбиение на группы сделано ориентировочно, и для окончательных выводов требуется более тщательный анализ материала.

Возникает вопрос, каким образом ложатся точки, насколько случайны их положения и какова роль ограничения их числа. Для этого мы с помощью генератора случайных чисел сформировали модельный набор 51 пар точек с близкими к экспериментальным значениям параметров. В основу была положена гауссова форма плотности вероятности попадания точки в тот или иной интервал. По оси абсцисс и по оси ординат были взяты 2 различные выборки по 51 точке и объединены в пары. Затем в частотных координатах был взят наш реальный центр и прямая с угловым наклоном, аппроксимирующая всю совокупность экспериментальных точек. Туда были вставлены пары точек, где осью абсцисс была аппроксимирующая прямая, а осью ординат перпендикуляр к ней. Эту совокупность будем называть случайной моделью. Эти точки представлены на рис. $2, b$. Угловой коэффициент общей совокупности 51 точки этой модели составил -0.208 . В подписи к рисунку приведено уравнение аппроксимирующей прямой. Затем было построено упорядоченное распределение по величине расстояний до этой прямой всей совокупности модельных точек. Оно показано в виде кривой 4 на рис. 3. Также была сформирована совокупность 510 случайных чисел с той же дисперсией, что и для совокупности модельных точек, для сопоставления с остальными данными (большая совокупность) и построено ее упорядоченное по значениям точек распределение. На рис. 3 кривые 3 (сплошные линии) демонстрируют это распределение для большой совокупности (510 точек), а кривые 4 для случайной модели (51 точка). Большая совокупность идет с довольно малыми шумами. Точки случайной модели идут в окрестности большой совокупности, не слишком удаляясь от нее (менее $100 \mathrm{~cm}^{-2}$ в интервале от точки 3 до точки 48). На ней имеется один достаточно заметный горизонтальный участок около точек 41-42. Между точками 13 и 14 наблюдается заметный перепад уровней около $100 \mathrm{~cm}^{-2}$. Однако в случайной модели не наблюдается ничего похожего на появление пустой области. Тем не менее некоторый эффект структурированности случайной модели в силу ограниченности выборки существует. По колебаниям упорядоченного распределения мы разбили точки модели на 7 групп и аппроксимировали прямыми линиями. Результат показан на рис. 2, $b$. Они вобрали все точки, кроме четырех крайних. Однако все линии расположены без заметных сгущений и разрежений. В целом характер поведения случайной выборки иной, нежели для экспериментальных точек.
В частности, различается статистика размеров интервалов. Если для экспериментальных точек интервалы групп короче и в пределы интервалов попало 38 точек, то для случайной модели интервалы групп длиннее и в пределы интервалов попало 47 точек из 51. Здесь все интервалы соприкасаются. В интервалы не вошли лишь 4 точки на границах области. Для экспериментальных же точек между несколькими интервалами имеются точки, не вошедшие в интервалы. Таким образом, характер распределения точек экспериментальных и модельных различен.

\section{7. Плотность вероятности в распределении точек}

Можно провести анализ неоднородности распределения точек в области, занимаемой ими, с помощью вычисления вероятности попадания точек в ту или иную область. Достаточно очевидно, что после разбиения на группы можно определить плотность числа точек как число точек, деленное на ширину интервала, в котором они располагаются. Это будет средняя плотность числа точек на этом интервале.

Здесь мы представляем результаты оценки вероятности с помощью описанного выше понятия плотности вероятности попадания точки в полосу. Она определяется с помощью вычисления производной расстояния от точки до опорной прямой по номеру точки упорядоченного распределения и позволяет оценивать неоднородность распределения точек внутри интервала. Для этих расчетов распределение расстояний до линии с наклоном 0.149 по характеру его поведения, описанному в предыдущем разделе, было разбито на 7 групп. В пределах одной группы соответствующий отрезок распределения аппроксимировался с помощью метода наименьших квадратов полиномом до 5-7-й степени со стабилизацией по всем производным. Далее вычислялась первая производная этого полинома, и результат деления единицы на эту производную после процедуры нормировки для всей совокупности интервалов разбиения принимался за плотность вероятности. Это позволяет судить не только о средней плотности вероятности в различных интервалах, но и о локальной плотности вероятности в пределах одного интервала. Результаты для экспериментальных точек показаны на рис. 4, $a$, а для случайной модели - на рис. $4, b$.

Результаты в основном соответствуют результатам, полученным в разд. 6. Для экспериментальных точек показаны вычисленные плотности вероятности, полученные для направления 0.149 (кривая 1) и 0.183 (кривая 2). Видно, что для групп с 1 по 3 (точки с 2 по 13, расстояния от прямой от $760 \mathrm{~cm}^{-2}$ до $450 \mathrm{~cm}^{-2}$ ) вероятности существенно больше, чем для других групп. При этом она максимальна для 3-й группы и внутри нее изменяется неоднородно. Также максимальна плотность и для 7-й группы (точки 41-44, расстояния от $-350 \mathrm{~cm}^{-2}$ до $-400 \mathrm{~cm}^{-2}$ ). Для расстояний от прямой с 

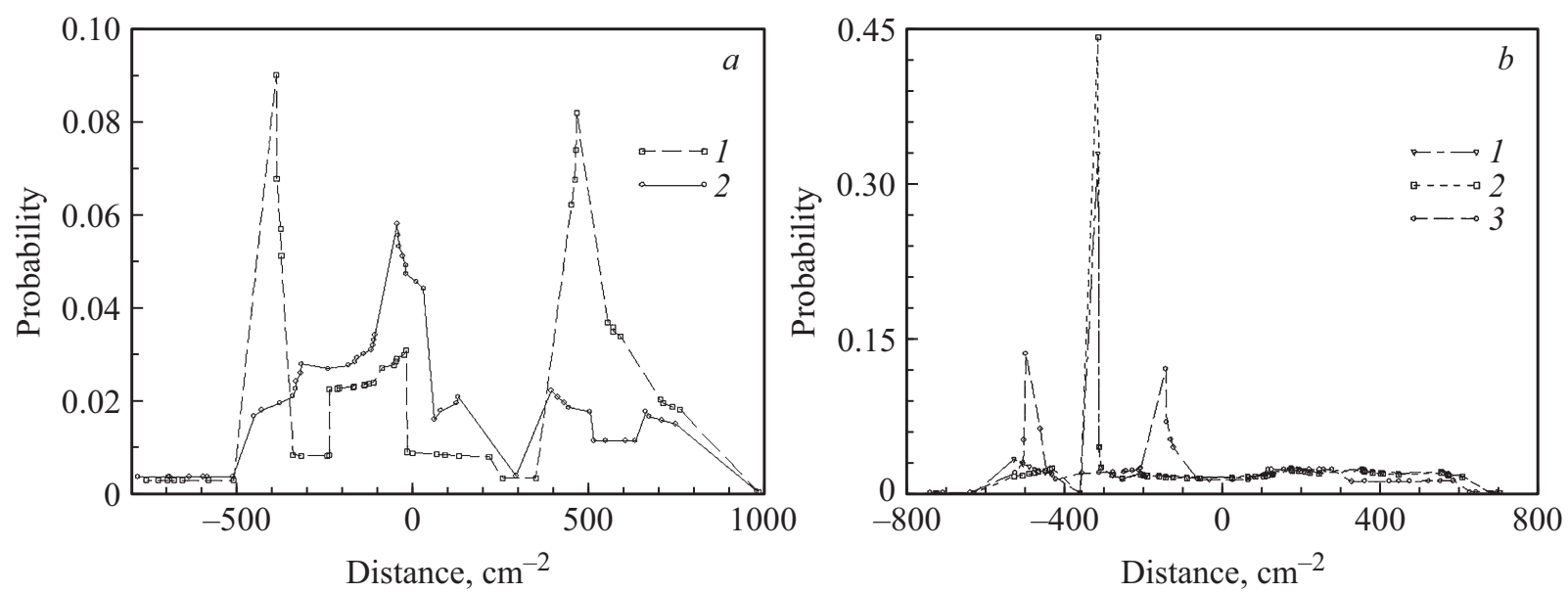

Рис. 4. Плотности вероятности в зависимости от расстояния до прямых с угловыми коэффициентами: $(a)$ для экспериментальных точек и угловых коэффициентов $-0.149(1),-0.183(2) ;(b)$ для точек случайной модели и угловых коэффициентов $-0.149(1)$, $-0.183(2),-0.208$ (3). Положительные значения расстояний на оси абсцисс соответствуют меньшим номерам в упорядоченной по расстояниям последовательности на рис. 3 и верхним точкам на рис. 2 , $a$.

наклоном 0.183 плотность вероятности для этих групп мала, но максимальна для двух групп, расположенных в центре около 0 (группы точек 15-21 и 22-27, кривая 1, кружки). Для первой из этих групп распределение сильно неоднородно в пределах группы.

На рис. 4, $b$ для случайной модели показаны данные для плотностей вероятностей, построенные для трех прямых с различными угловыми коэффициентами. Здесь обращает на себя внимание очень узкий резонансный пик одной точки для угловых коэффициентов -0.149 и -0.183 , который максимален для последней прямой. Для прямой с коэффициентом -0.208 этот резонансный пик отсутствует, но появляются 2 симметричных более широких и более низких пика, почти равноотстоящих от исчезнувшего пика. Эта искусственная модель отличается и более пониженным и протяженным уровнем остального сигнала по сравнению с экспериментальными точками. В частотной области между выделенными группами, точки каждой из которых аппроксимировались прямой, эти группы в отличие от экспериментальных точек своими границами примыкали друг к другу достаточно близко. Таким образом, влияние ограниченной выборки может приводить к ложной структуризации данных в спектральных координатах, но характеры поведения случайных модельных точек и экспериментальных различаются. В частности, практически нет сближения более чем двух точек, что наблюдается для воды.

\section{8. Структуризация распределения точек в спектральных координатах}

Подчеркнем, что структуризация распределения точек, связанная с наличием области с пониженной вероятностью появления точек, носит объективный характер и проявляется во всех проведенных нами экспериментах и на данных для обоих колебаний. Они, несомненно, связаны со свойствами наших образцов. Параметры осцилляторов, полученные для каждой из областей, вполне однозначны и относятся к средним эффективным параметрам совокупностей данных.

Приведенные здесь данные более мелкомасштабных структурных элементов распределения точек в частотных координатах несомненно существуют. С чем связаны вызванные свойствами воды структурные особенности представленной картины, пока сказать трудно, скорее всего, давно обсуждающиеся в литературе проблемы структуры воды могут иметь к этому отношение [10,9-22,25-28].

Во всяком случае, видимо, так получаемые плотности вероятности могут помочь при объективном разбиении совокупности экспериментальных точек на группы, для которых можно определять параметры осцилляторов.

\section{Выводы}

1. В работе представлены спектральные параметры низкочастотных $\left(50 \mathrm{~cm}^{-1}\right)$ и высокочастотных $\left(184 \mathrm{~cm}^{-1}\right)$ колебательных компонент динамической восприимчивости воды, полученных для 51 низкочастотного спектра КРС.

2. Данные для каждого из колебаний представлены в частотных координатах квадрат наблюдаемой ширины - квадрат частоты соответствующего лоренциана, и получены совокупные эффективные параметры соответствующих осцилляторов: собственные частоты, коэффициенты неоднородности. Эти параметры хорошо совпали с совокупными параметрами, полученными для совокупности 7 спектров воды, опубликованных нами ранее [1].

3. Было обнаружено, что в этих координатах параметры обоих осцилляторов разбиваются на 2 области, разделенные практически незаполненной точками областью с малой вероятностью появления точек. 
4. Для высокочастотного колебания были определены эффективные параметры осцилляторов для совокупностей точек этих областей: собственные частоты и коэффициенты неоднородности. Коэффициенты неоднородности для этих областей практически совпали друг с другом, но отличаются от соответствующей величины для общей совокупности всех точек, а собственные частоты различаются на $2.2 \mathrm{~cm}^{-1}$, при этом верхняя из них практически совпала с ее значением для всей совокупности.

5. Обнаружено наличие более мелкомасштабной значительной неоднородности распределения данных в частотных координатах, причина которых пока осталась не выясненной.

\section{Список литературы}

[1] Kraiskii A.V., Mel'nik N.N. // Opt. Spectrosc. 2018. V. 124. N 5. P. 660. doi 10.21883/OS.2018.05.45943.300-17; Крайский А.В., Мельник Н.Н. // Опт. и спектр. 2018. Т. 124. № 5. C. 628.

[2] Крайский А.В., Мельник Н.Н. // Краткие сообщения по физике ФИАН. 2005. № 12. С. 26; Kraiski A.V., Mel'nik N.N. // Bulletin of the Lebedev Physics Institute. 2005. V. 32. N 12. P. 21.

[3] Крайский А.В., Мельник Н.Н. // Биофизика. 2012. Т. 57. № 6. C. 965; Kraiski A.V., Mel'nik N.N. // Biophysics. 2012. V. 57. N 6. P. 750.

[4] Shuker R., Gammon R.W. // J. Chem. Phys. 1971. V. 55. P. 4784.

[5] Bucaro J.A., Litovitz T.A. // J. Chem. Phys. 1971. V. 55. P. 3585.

[6] Lund P.A., Nielsen O.F., Praestgaard E. // Chem. Phys. 1978. V. 28. P. 167.

[7] Nielsen O.F., Christensen D.H., Lund P.A., Praestgaard E. // Proceedings of the 6th International Conference on Raman Spectroscopy. Bangalore, India. Heyden, London, 1978. V. 2. P. 208.

[8] Walrafen G.E., Chu Y.C., Carlon H.R. Proton Transfer in Hydrogen-bonded Systems. / Ed. by Bountis T. New York: Plenum Press, 1992.

[9] Yuko Amo, Yasunori Tominaga. // Physica A. 2000. V. 276. P. 401.

[10] Brooker M.H., Nielsen O.F., Praestgaard E. // J. Raman Spectrosc. 1988. V. 19. P. 71.

[11] Walrafen G.E., Chu Y.C., Piermarini G.J. // J. Phys. Chem. 1996. V. 100. P. 10363.

[12] Chaplin M. Электронный ресурс. Режим доступа: http://www.lsbu.ac.uk/water/vibrat.html Water Absorption Spectrum, in http://www.lsbu.ac.uk/water/index2.html Water Structure and Science

[13] Tsai K.H., Wu T.-M. // Chem. Phys. Lett. 2006. V. 417. P. 389-394.

[14] Gaiduk V.I., Crothers D.S.F. // J. Mol. Liq. 2006. V. 128. P. $145-160$

[15] Гайдук В.И. // Опт. и спектр. 2009. Т. 106. № 1. С. 28-46.

[16] Mizoguchi K., Hori Y., Tominaga Y. // J. Chem. Phys. 1992. V. 97. P. 1961.

[17] Winkler K., Lindner J., Vohringer P. // Phys. Chem. Chem. Phys. 2002. V. 4. P. 2144.
[18] Idrissi A., Longelin S., Sokolič F. // J. Phys. Chem. B. 2001. V. 105. P. 6004.

[19] De Santis A., Erocli A., Rocca D. // J. Chem. Phys. 2004. V. 120. P. 1657.

[20] Padro J.A., Marti J. // J. Chem. Phys. 2003. V. 118. P. 452.

[21] Galvin M., Zerulla D. // Chem. Phys. Chem. 2011. V. 12. N 5. P. 913-914.

[22] Gaiduk V.I., Nielsen O.F., Crothers D.S.F. // J. Molec. Liquids. 2008. V. 137. P. $92-103$.

[23] Крайский А.В., Мельник Н.Н. // Краткие сообщения по физике. М.: ФИАН, 2006. № 1. С. 42; Kraiski A.V., Mel'nik N.N. // Bulletin of the Lebedev Physics Institute. Moscow, 2006. V. 33. N 1. P. 30.

[24] Крайский А.В., Мельник Н.Н. // Краткие сообщения по физике. ФИАН, 2006. № 1. С. 49; Kraiski A.V., Mel'nik N.N. // Bulletin of the Lebedev Physics Institute. 2006. V. 33. N 1. P. 34.

[25] Маленков Г.Г. // Журнал структурной химии. 2007. Т. 48. № 4. C. 772.

[26] Першин С.М. // Биофизика. 2014. Т. 59. № 6. С. 1209.

[27] Захаров С.Д. // Биофизика. 2012. Т. 57. № 6. С. 1041.

[28] Першин С.М., Леднёв В.Н., Фёдоров А.Н. // Краткие сообщения по физике. ФИАН, 2018. Т. 45. № 2. С. 3. 